\title{
Farey fractions with prime denominators
}

by

\author{
D. E. WelCH (Cheltenham)
}

1. Introduction. The following problem was suggested by my supervisor Professor Huxley for the submission of my PhD thesis, [5].

Let $\mathcal{F}(Q)$ be the Farey sequence of order $Q$ and $\alpha$ a real number in the interval $(0,1)$. If we travel from $\alpha$ along the real line in either direction, how many Farey fractions do we need to pass to ensure that we have passed one which has prime denominator?

2. The theorem. Let $N_{Q}(\alpha, \beta)$ be the number of fractions of $\mathcal{F}(Q)$ in the interval $[\alpha, \beta]$ (if $\alpha \leq \beta$ ) or $[\beta, \alpha]$ (if $\beta \leq \alpha$ ). Then the problem is equivalent to obtaining an upper bound to

$$
\min _{p \leq Q} N_{Q}(\alpha, a / p)
$$

for $p$ a prime. Trivially we have

$$
\min _{p \leq Q} N_{Q}(\alpha, a / p) \ll Q
$$

just from the contribution of the largest prime less than $Q$.

We use Fogels' reformulation of Linnik's theorem on primes in short segments of arithmetic progressions and a special case of Vaughan's theorem on the distribution of $\alpha p$ modulo one to obtain the following theorem.

THEOREM 2.1. Let $\alpha$ be a real number in the interval $(0,1)$. Then there exists a Farey fraction $a / p$ such that $p \in[Q / 2, Q]$ is prime and

$$
N_{Q}(\alpha, a / p) \ll Q^{1-1 / 1312} \log ^{8} Q .
$$

The proof of Theorem 2.1 depends on whether $\alpha$ is in the major or minor arcs case.

2000 Mathematics Subject Classification: Primary 11M99.

Key words and phrases: Farey fractions with prime denominators, Linnik's theorem, Fogels' theorem, primes in short arithmetic progressions, approximation theory. 
3. The minor arcs case. In the minor arcs case we can approximate $\alpha$ by a Farey fraction with prime denominator using Vaughan's result from the $\|\alpha p\|$ problem, and then bound the number of Farey fractions that can lie between $\alpha$ and our approximation with the following lemma taken from Huxley [2].

Lemma 3.1. Let I be an interval of length $\Delta$. Then

$$
\sum_{a / q \in \mathcal{F}(Q) \cap I} 1 \leq \Delta Q^{2}+1
$$

Vaughan's result on the distribution of $\alpha p$ modulo one (see [3]) is a corollary of the following theorem which we will require.

Theorem 3.2. Suppose that $(a, q)=1,|\alpha-a / q| \leq 1 / q^{2}, H \geq 1, N \geq 1$. Let

$$
S=\sum_{h \leq H}\left|\sum_{n \leq N} \Lambda(n) e(\alpha h n)\right|,
$$

where $\Lambda$ is von Mangoldt's function, and $e(x)=\exp (2 \pi i x)$. Then

$$
S \ll H N \log ^{8} H N\left(\frac{1}{q^{1 / 2}}+\frac{1}{N^{1 / 4}}+\left(\frac{q}{H N}\right)^{1 / 2}+\left(\frac{1}{H^{2} N}\right)^{1 / 5}\right) .
$$

We use Theorem 3.2 to prove the following corollary.

Corollary 3.3. Suppose $\alpha$ is a real number in $(0,1), Q$ is a large positive integer and $a / q$ is a convergent to the continued fraction expansion for $\alpha$ with $q$ in the range

$$
\frac{\log ^{16} Q}{\delta^{2}} \ll q \ll \frac{\delta Q}{\log ^{16} Q},
$$

where

$$
\frac{\log ^{8} Q}{Q^{1 / 4}} \ll \delta<\frac{1}{2} .
$$

Then there is a prime $p \in[Q / 2, Q]$ for which $\|\alpha p\| \ll \delta$.

Proof. Let $\varrho(t)=[t]-t+1 / 2$ be the rounding error function, which is well known to have Fourier expansion

$$
\varrho(t)=\sum_{1 \leq|h| \leq H} \frac{e(h t)}{2 \pi i h}+O\left(\min \left(1, \frac{1}{H\|t\|}\right)\right),
$$

for $t$ not an integer. Then for $\delta<1 / 2$, the sum

$$
\sum_{Q / 2<p \leq Q}(\varrho(\alpha p+\delta)-\varrho(\alpha p-\delta)+2 \delta)
$$

counts the exact number of primes in the range $(Q / 2, Q]$ for which $\|\alpha p\|<\delta$. Following Vaughan we count powers of primes with a logarithmic weight and 
so work with the sum

$$
\sum_{\substack{Q / 2<n \leq Q \\\|\alpha n\|<\delta}} \Lambda(n)=\sum_{\substack{Q<2<n \leq Q \\ \text { ind }}} \Lambda(n)(\varrho(\alpha n+\delta)-\varrho(\alpha n-\delta)+2 \delta) .
$$

By the prime number theorem the right hand side of (3.2) reduces to

$$
2 \delta Q+\Sigma_{1}+O\left(\frac{\delta Q}{\log Q}\right),
$$

where

$$
\Sigma_{1}=\sum_{Q / 2<n \leq Q} \Lambda(n)(\varrho(\alpha n+\delta)-\varrho(\alpha n-\delta)) .
$$

We estimate $\Sigma_{1}$ using (3.1), which gives

$$
\Sigma_{1}=\sum_{Q / 2<n \leq Q} \Lambda(n)\left(\sum_{1 \leq|h| \leq H} \frac{e(h(\alpha n+\delta))-e(h(\alpha n-\delta))}{2 \pi i h}\right)+O\left(\left|\Sigma_{2}\right|\right),
$$

where

$$
\begin{aligned}
\Sigma_{2} & =\frac{1}{H} \sum_{n \leq Q} \Lambda(n) \min \left(H, \frac{1}{\|\alpha n+\delta\|}\right) \\
& \leq \frac{\log Q}{H}\left(1+\frac{Q}{q}\right)(3 H+4 q \log H),
\end{aligned}
$$

by Lemmas 8a and 8b of Chapter 1 of Vinogradov [4]. We choose $H=\delta^{-1}$, so that the right hand side of (3.4) is

$$
\leq 3\left(1+\frac{Q}{q}\right) \log Q .
$$

Now

$$
\begin{aligned}
\Sigma_{1}+O & \left(\frac{Q \log Q}{q}\right) \\
& =\sum_{q / 2<n \leq Q}\left(\sum_{1 \leq|h| \leq H} \frac{\Lambda(n) e(\alpha h n)}{2 \pi i h}(e(h \delta)-e(-h \delta))\right) \\
& =\sum_{q / 2<n \leq Q}\left(\sum_{1 \leq|h| \leq H} \frac{\Lambda(n) e(\alpha h n)}{2 \pi i h} \int_{-\delta}^{\delta} 2 \pi i h e(h t) d t\right) \\
& \ll \delta \sum_{h \leq H}\left|\sum_{Q / 2<n \leq Q} \Lambda(n) e(\alpha h n)\right| \\
& \ll \delta H Q \log ^{8}(H Q)\left(\frac{1}{q^{1 / 2}}+\frac{1}{Q^{1 / 4}}+\left(\frac{q}{H Q}\right)^{1 / 2}+\left(\frac{1}{H^{2} Q}\right)^{1 / 5}\right)
\end{aligned}
$$


by Theorem 3.2. Thus we have

$$
\Sigma_{1} \ll Q \log ^{8} Q\left(\frac{1}{q^{1 / 2}}+\frac{1}{Q^{1 / 4}}+\left(\frac{\delta q}{Q}\right)^{1 / 2}+\left(\frac{\delta^{2}}{Q}\right)^{1 / 5}\right),
$$

which we require to be $\ll \delta Q$ which yields the conditions on $q$ and $\delta$ stated in Corollary 3.3 and the result follows.

4. The major arcs case. In the major arcs case, denominators of nearby Farey fractions form short segments of arithmetic progressions within which we require a prime number near $\alpha$. We use the following theorem due to Fogels [1].

THEOREM 4.1. There exist constants $\theta \in(0,1)$ and $c>1 / \theta$ such that for $q$ large enough and any $x>q^{c}$ the interval $\left(x, x+x^{\theta}\right)$ contains a prime $p \equiv l \bmod q$ as long as $(q, l)=1$.

Fogels outlines the proof of Theorem 4.1 as an application of his Linnik type zero density theorem which extends the range of zeros of Dirichlet $L$-functions previously considered by Linnik and others. Fogels does not provide admissible values for $\theta$ and $c$ presumably due to the complicated nature of the proof of his zero density theorem. Since then there have been many developments in the proof of Linnik's theorem which make calculation of the constants involved a great deal simpler. Welch [5] proves Theorem 4.1 with $c=328$ and $\theta=655 / 656$.

We begin the major arcs case by extending the result on $\|\alpha p\|$ to a larger range for $\alpha$. However, when the difference between denominators in the convergents of $\alpha$ becomes too large we must resort to counting Farey fractions directly without recourse to approximations of $\alpha$ involving prime denominators.

4.1. The major arcs case 1. We prove the following lemma.

Lemma 4.2. Suppose that $\alpha$ is a real number in the interval $(0,1)$ with two successive convergents in its continued fraction expansion $a_{r} / q_{r}$ and $a_{r+1} / q_{r+1}$, where

$$
\begin{aligned}
q_{r} & \ll \frac{\log ^{16} Q}{\delta^{2}}, \\
\frac{\delta Q}{\log ^{16} Q} & \ll q_{r+1}<Q .
\end{aligned}
$$

Then there is a prime number $p \in(q / 2, Q]$ for which $\|\alpha p\| \ll \delta$.

Proof. For simplicity we shall assume that $r$ is even; the proof when $r$ is odd follows similarly. 
Since $a_{r} / q_{r}$ and $a_{r+1} / q_{r+1}$ are succesive convergents of $\alpha$, we have

$$
\frac{a_{r}}{q_{r}}<\alpha<\frac{a_{r+1}}{q_{r+1}} .
$$

Farey fractions of $\mathcal{F}(Q)$ in the interval $\left(a_{r} / q_{r}, a_{r+1} / q_{r+1}\right)$ are of the form

$$
\frac{a_{r} x+a_{r+1} y}{q_{r} x+q_{r+1} y},
$$

where $(x, y)=1$, and $q_{r} x+q_{r+1} y \leq Q$. If we now fix $y=y_{0}$ so that

$$
Q / 2 \leq q_{r+1} y_{0}<Q,
$$

and allow $x$ to vary subject to the constraints $\left(x, y_{0}\right)=1$ and $q_{r} x+q_{r+1} y_{0}$ $\leq Q$, we have a short arithmetic progression within which we require a prime. Inequality (4.3) will ensure that the prime is of the correct size. However, as $x$ increases we could be moving too far away from $\alpha$ to ensure a sufficient approximation. Thus we wish to bound $x$ in such a way that

$$
\left|\alpha-\frac{a_{r} x+a_{r+1} y_{0}}{q_{r} x+q_{r+1} y_{0}}\right| \ll \frac{\delta}{Q},
$$

so that we can satisfy the inequality $\|\alpha p\| \ll \delta$.

We first consider the case where

$$
\frac{a_{r} x+a_{r+1} y_{0}}{q_{r} x+q_{r+1} y_{0}} \in\left(\frac{a_{r}}{q_{r}}, \alpha\right) .
$$

We have the following inequality:

$$
\begin{aligned}
\left|\alpha-\frac{a_{r} x+a_{r+1} y_{0}}{q_{r} x+q_{r+1} y_{0}}\right| & \leq\left|\frac{a_{r+1}}{q_{r+1}}-\frac{a_{r} x+a_{r+1} y_{0}}{q_{r} x+q_{r+1} y_{0}}\right| \leq \frac{x}{q_{r+1}\left(q_{r} x+q_{r+1} y_{0}\right)} \\
& \leq \frac{2 x}{q_{r+1} Q} \ll \frac{x \log ^{16} Q}{\delta Q^{2}},
\end{aligned}
$$

by (4.2). In order to satisfy (4.4) we require the following bound on $x$ :

$$
x \ll \frac{\delta^{2} Q}{\log ^{16} Q} .
$$

Now suppose that

$$
\frac{a_{r} x+a_{r+1} y_{0}}{q_{r} x+q_{r+1} y_{0}} \in\left(\alpha, \frac{a_{r+1}}{q_{r+1}}\right) .
$$

Then we have

$$
\left|\alpha-\frac{a_{r} x+a_{r+1} y_{0}}{q_{r} x+q_{r+1} y_{0}}\right| \leq\left|\alpha-\frac{a_{r+1}}{q_{r+1}}\right| \leq \frac{1}{q_{r+1}^{2}} \ll \frac{\log ^{32} Q}{\delta^{2} Q^{2}},
$$

by (4.2). Therefore in order to satisfy (4.4) we also require

$$
\left(\frac{\log ^{32} Q}{Q}\right)^{1 / 3} \ll \delta
$$


So the denominators of our subsequence of the Farey sequence form an arithmetic progression $l \bmod q_{r}$ of length $L$, where

$$
L \asymp \frac{\delta^{2} Q}{\log ^{16} Q},
$$

by (4.5). Theorem 4.1 states that this arithmetic progression will contain a prime number if $q_{r}^{328} \leq Q$ and $L \ll Q^{655 / 656}$. Both conditions imply the following lower bound for $\delta$ :

$$
\delta \gg \frac{\log ^{8} Q}{Q^{1 / 1312}},
$$

which is consistent with the inequality (4.6) and thus completes the proof of Lemma 4.2 .

4.2. The major arcs case 2. We need now to consider the case where either $q_{r+1} \geq Q$ or there is no $q_{r+1}$, i.e. $\alpha=a_{s} / q_{s}$ for some $s \leq r$. This is where estimates of $\|\alpha p\|$ are of no advantage since there are not enough Farey fractions near $\alpha$. We prove the following lemma.

LEMMA 4.3. If $\alpha$, a real number in $(0,1)$, has a convergent $a_{r} / q_{r}$ such that it is the best approximation to $\alpha$ in $\mathcal{F}(Q)$ and

$$
q_{r} \ll \frac{\log ^{16} Q}{\delta^{2}},
$$

then the interval $(\alpha, \beta)$ or $(\beta, \alpha)$ contains a Farey fraction a/p of $\mathcal{F}(Q)$, where $p$ is a prime number and

$$
N_{Q}(\alpha, \beta) \ll \delta Q
$$

for

$$
\delta \gg \frac{\log ^{8} Q}{Q^{1 / 1312}} .
$$

Proof. Instead of looking at Farey fractions between $a_{r} / q_{r}$ and $a_{r+1} / q_{r+1}$ as we did in the previous section, we now concentrate on Farey fractions between $a_{r} / q_{r}$ and $a_{r-1} / q_{r-1}$. Consider the sequence of rationals

$$
f(x)=\frac{a_{r} x+a_{r-1}}{q_{r} x+q_{r-1}},
$$

which is tending towards the nearest fraction of $\mathcal{F}(Q)$ to $a_{r+1} / q_{r+1}$. We look to the interval $\left(f\left(x_{0}\right), f(X)\right)$ for our Farey fraction with prime denominator, where

$$
Q / 2 \leq q_{r} x_{0}+q_{r-1} \leq 3 Q / 4 \text { and } q_{r} X+q_{r-1} \leq Q
$$

The distance between these two rationals is given by

$$
\left|\frac{a_{r} X+a_{r-1}}{q_{r} X+q_{r-1}}-\frac{a_{r} x_{0}+a_{r-1}}{q_{r} x_{0}+q_{r-1}}\right|=\frac{\left|X-x_{0}\right|}{\left(q_{r} X+q_{r-1}\right)\left(q_{r} x_{0}+q_{r-1}\right)} \ll \frac{\left|X-x_{0}\right|}{Q^{2}} \ll \frac{\delta}{Q}
$$


for $X \asymp \delta Q$, from which it follows that

$$
N_{Q}\left(f\left(x_{0}\right), f(X)\right) \ll \delta Q
$$

However, we require an estimate for $N_{Q}\left(\alpha, f\left(x_{0}\right)\right)$ :

$$
N_{Q}\left(\alpha, f\left(x_{0}\right)\right)=N_{Q}(\alpha, f(X))+N_{Q}\left(f(X), f\left(x_{0}\right)\right),
$$

where

$$
\begin{aligned}
N_{Q}(\alpha, f(X)) & =N_{Q}\left(a_{r+1} / q_{r+1}, f(X)\right)+N_{Q}\left(\alpha, a_{r+1} / q_{r+1}\right) \\
& =N_{Q}\left(a_{r+1} / q_{r+1}, f(X)\right),
\end{aligned}
$$

since $N_{Q}\left(\alpha, a_{r+1} / q_{r+1}\right)=0$. We have

$$
\begin{aligned}
\left|\frac{a_{r+1}}{q_{r+1}}-f(X)\right| & =\left|\frac{a_{r+1}}{q_{r+1}}-\frac{a_{r} X+a_{r-1}}{q_{r} X+q_{r-1}}\right|=\left|\frac{X+a_{r+1} q_{r-1}+a_{r-1} q_{r+1}}{q_{r+1}\left(q_{r} X+q_{r-1}\right)}\right| \\
& \ll \frac{X}{Q^{2}} \ll \frac{\delta}{Q},
\end{aligned}
$$

and so (4.8) and (4.9) give

$$
N_{Q}\left(\alpha, f\left(x_{0}\right)\right) \ll \delta Q .
$$

The fact that there is a Farey fraction with prime denominator in the interval $\left(f\left(x_{0}\right), f(X)\right)$ follows from the major arcs case 1 by considering the arithmetic progression $q_{r} x+q_{r-1}$.

5. Proof of the theorem. Theorem 2.1 now follows from Lemma 4.3 and by using Lemma 3.1 in conjunction with Corollary 3.3 and Lemma 4.2.

\section{References}

[1] E. Fogels, On the zeros of L-functions, Acta Arith. 11 (1965), 67-96.

[2] M. N. Huxley, Area, Lattice Points and Exponential Sums, Clarendon Press, Oxford, 1996.

[3] R. C. Vaughan, On the distribution of ap modulo 1, Mathematika 24 (1977), 135-141.

[4] I. M. Vinogradov, The Method of Trigonometrical Sums in the Theory of Numbers, translated from the Russian, revised and annotated by K. F. Roth and A. Davenport, Interscience, London, 1954.

[5] D. E. Welch, Some problems involving prime numbers, $\mathrm{PhD}$ thesis, 2007.

24 Reddings Park

Cheltenham, Glos, GL51 6UD, UK

E-mail: wdazaman@aol.com

Received on 26.9.2007

and in revised form on 13.4.2008 